Measurement and modeling of dispersive pulse propagation in drawn wire waveguides

\author{
Eric I. Madaras \\ NASA Langley Research Center, Hampton, Virginia 23681-0001 \\ Thomas W. Kohl and Wayne P. Rogers \\ University of Colorado, Department of Mechanical Engineering, Boulder, Colorado 80309-0427
}

(Received 20 October 1993; accepted for publication 12 July 1994)

\begin{abstract}
An analytical model of dispersive pulse propagation in semi-infinite cylinders due to transient axially symmetric end conditions has been experimentally investigated. Specifically, the dispersive propagation of the first axially symmetric longitudinal mode in thin wire waveguides, which have ends in butt contact with longitudinal piezoelectric ultrasonic transducers, is examined. The method allows for prediction of a propagated waveform given a measured source waveform, together with the material properties of the cylinder. Alternatively, the source waveform can be extracted from measurement of the propagated waveform. The material properties required for implementation of the pulse propagation model are determined using guided wave phase velocity measurements. Hard tempered aluminum 1100 and 304 stainless steel wires, with 127, 305, and $406 \mu \mathrm{m}$ diam, were examined. In general, the drawn wires were found to behave as transversely isotropic media.
\end{abstract}

PACS numbers: 43.35.Pt, 43.20.Jr

\section{INTRODUCTION}

Historically, pulse propagation in thin cylindrical waveguides has been of interest for applications including the material testing of wires and fibers, ${ }^{1-3}$ ultrasonic delay lines, ${ }^{4}$ medical ultrasonics, ${ }^{5}$ and embedded sensor materials. ${ }^{6}$ Propagation of ultrasonic waves in cylinders has been the subject of several investigations, both analytical and experimental. ${ }^{7-20}$ In high-frequency and broadband applications, these cylinders may exhibit considerable dispersion when an acoustic pulse propagates through the waveguide. Here, dispersion is the dependence of acoustic guided wave phase velocity on frequency, resulting in distortion of the waveform of a transient plane-wave pulse depending on the axial location. This pulse distortion can make the interpretation of measured waveforms difficult. The present paper combines experimental measurements and analytical modeling to yield a quantitative and integrated approach to address the problem of pulse dispersion in cylindrical waveguides. The method presented also has significant relevance to the problem of material characterization of these waveguides.

\section{PULSE PROPAGATION}

While exact ${ }^{7-9}$ and approximate ${ }^{10,11}$ solutions have been used to study the axially symmetric guided wave phase velocity versus frequency relationship in solid cylindrical waveguides, and experimental dispersion measurements have corroborated the theory, ${ }^{12-15}$ few studies have been done to assess the capability these solutions yield for modeling actual pulse propagation. The earliest analytical solution, using exact theory, to the problem of axially symmetric pulse propagation in a cylindrical waveguide due to application of a transient end condition can be attributed to Skalak. ${ }^{16}$ A special solution was obtained to the problem of a solid isotropic cylinder with a velocity imparted uniformly to the cylinder end in the form of a step function. Subsequently, Folk et al. ${ }^{17}$ used exact theory to obtain a solution for a solid isotropic cylinder with arbitrary time harmonic mixed (combined displacement and stress) end boundary conditions. Moreover, an attempt was made by Folk et al. ${ }^{17}$ to experimentally validate the solution in terms of its ability to model pulse propagation. Studies by Miklowitz and Nisewanger, ${ }^{18}$ Hseih and Kolsky, ${ }^{19}$ and McNiven and Mengi ${ }^{20}$ have attempted to experimentally validate propagation models based on approximate theories. Only qualitative agreement between theoretical and measured waveforms was obtained in these studies. Furthermore, the pulses examined in all of these studies were dominated by lower-frequency propagation of the first longitudinal mode. The pulses, resulting from either ideal or modified (some finite rise time) step loading, essentially consist of a low-frequency leading head and a higher-frequency dispersive tail. While the current study also addresses first longitudinal mode propagation, the transient end conditions applied give rise to more varied dispersive pulse distortion. The frequency range considered extends from low frequency, where the fundamental tends toward nondispersiveness, to approaching the second longitudinal mode cutoff, where the fundamental becomes highly dispersive. An integrated analytical and experimental study of isotropic hollow cylinders using the same numerical method and similar measurement techniques ${ }^{21}$ involves the propagation of higher-order longitudinal modes.

\section{PROPERTY MEASUREMENT}

Because the dispersion of the guided waves depends on the elastic properties, cylindrical guided wave propagation has been used for the material characterization and testing of wires and fibers. Meeker and Meitzler ${ }^{22}$ suggested several 
methods for determining material properties by measuring acoustic guided wave velocities, and a thorough review of the subject has been given by Curtis.'

Principally, velocity measurements of the first torsional mode and the long wavelength limit of the first longitudinal mode have been used to obtain the shear and Young's moduli, respectively, for isotropic cylinders. ${ }^{1,2}$ The first axisymmetric torsional mode is nondispersive, propagating at the shear wave velocity $(G / \rho)^{1 / 2}$. In the long wavelength (low-frequency) limit, the velocity of the first longitudinal axisymmetric mode tends toward the bar velocity $(E / \rho)^{1 / 2}$. As the limit is approached, group and phase velocities tend toward a constant and equal value, indicating nondispersive behavior.

However, the drawing process in the manufacture of wires and fibers can affect the material microstructure, frequently causing the material to be anisotropic.' Transverse isotropy, with five independent elastic constants, is considered to better represent the state of the material than isotropy. The five independent elastic properties for transversely isotropic media are taken as $E_{\mathrm{LL}}, E_{\mathrm{TT}}, G_{\mathrm{LT}}, G_{\mathrm{TT}}, \nu_{\mathrm{LT}}$, where $E, G$, and $\nu$ are Young's moduli, shear moduli, and poisson ratio, respectively, $L$ is longitudinal, and $T$ is transverse. ${ }^{23}$ Moreover, the propagation of the fundamental longitudinal mode, within the frequency range considered, does not depend on the transversely isotropic property to which the lowest torsional mode is sensitive, $G_{\mathrm{LT}}$. Rather, the strongest dependence is on $E_{\mathrm{LL}}$ and $\nu_{\mathrm{LT}}$.

Longitudinal mode measurement to obtain $E_{\mathrm{LL}}$ can be problematic. When the velocity of the long wavelength limit is used, a transient pulse with sufficient low-frequency content is propagated and its first arrival measured. Since the longest wavelengths of the first longitudinal mode propagate with greatest velocity, this time-of-flight measurement yields the velocity of the long wavelength limit. However, the leading head of a dispersive pulse often rises very gradually making accurate assessment of when the first arrival occurs difficult, as discussed by Curtis, ${ }^{1}$ and evident in the lowfrequency experimental waveforms of other studies. ${ }^{17-20}$ Consequently, the measurement may be subject to some error. Instead, the time shift between the peak of the wave envelope of a propagated broadband pulse is often matched with that of the initial pulse. ${ }^{2}$ However, the frequency and wavelength of such a velocity measurement is not at all clear. $^{24}$

Alternatively, phase or group velocity of particular cylindrical guided wave modes can be measured over a range of frequency. Material properties are then varied to obtain correspondence between theoretical velocities and the dispersion measurements. As noted by Meeker and Meitzler, ${ }^{22}$ dispersion measurements of the first axially symmetric longitudinal mode are sufficient to find both elastic constants for isotropic media. As will be illustrated here, combination of such measurement with measures of first torsional mode velocity yields $E_{\mathrm{LL}}, \nu_{\mathrm{LT}}$, and $G_{\mathrm{LT}}$ for transversely isotropic media. The extent of the material anisotropy can then be gauged.

Phase velocity measurement methods are preferred to group velocity measurement methods since the wave propa-

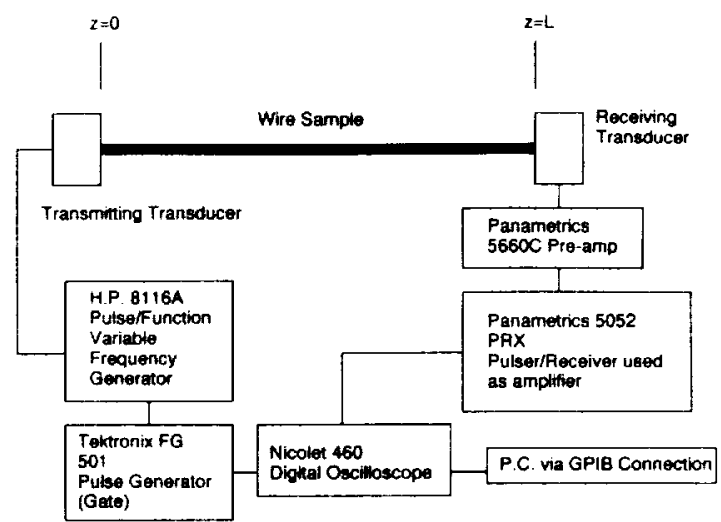

FIG. 1. Experimental apparatus.

gation is generally dispersive. Inherent difficulty in group velocity measurements for dispersive media has been noted. ${ }^{24.25}$ The problem stems from the distortion of the propagated waveform relative to the initially generated waveform and the resulting ambiguity in correlating the two different waveforms, and also, in determining the frequency for which the group velocity is being measured. Correspondingly, precision and accuracy for phase velocity mcasurement can be expected to exceed that obtained for group velocity measurement for dispersive pulse propagation. The scatter of group velocity measurements which commonly occurs for propagation in dispersive media can be seen in a recent study of sound in wires by Nicholson et al. ${ }^{15}$ The precision of the measurement and smoothness of the curve determined by these measurements is critical to accurate and repeatable material property characterization. The elastic properties determined from the phase velocity measurements are used here as input to the pulse propagation model.

\section{WAVE EXCITATION AND MEASUREMENT}

The method by which elastic pulses are launched into the wire specimens and measured after propagating the length of the sample is illustrated in Fig. 1. Similar techniques have been used in other studies of ultrasound propagation in wires. ${ }^{15}$ As shown in Fig. 1, matched pairs of piezoelectric longitudinal or shear wave transducers are placed in butt contact with each end of the wire and operate in pitch catch mode. For this study, $5 \mathrm{MHz}$ center frequency panametrics V109 (longitudinal) and V156 (shear) transducers were used. Glycerin was used as couplant to aid in sound transmission across the transducer/wire interface. The source or excitation transducer was provided a gated sine wave or toneburst electrical signal. Because of the thinness of the wires, the efficiency at which sound could be introduced into the waveguides was very low. Consequently, gain in the receiving system between 60 and $100 \mathrm{~dB}$ was required and signal averaging between 300 and 600 data records was performed to improve signal to noise. Signals were captured and averaged on a digital oscilloscope and processed on a $\mathrm{PC}$.

With longitudinal wave transducers in use, the pulse launched in the medium would be composed primarily of the first axially symmetric longitudinal mode. However, depending on factors such as the wire thickness, excitation fre- 


\title{
Measurement and modeling of dispersive pulse propagation in drawn wire waveguides
}

\author{
Eric I. Madaras \\ NASA Langley Research Center, Hampton, Virginia 23681-0001 \\ Thomas W. Kohl and Wayne P. Rogers \\ University of Colorado, Department of Mechanical Engineering, Boulder, Colorado 80309-0427
}

(Received 20 October 1993; accepted for publication 12 July 1994)

\begin{abstract}
An analytical model of dispersive pulse propagation in semi-infinite cylinders due to transient axially symmetric end conditions has been experimentally investigated. Specifically, the dispersive propagation of the first axially symmetric longitudinal mode in thin wire waveguides, which have ends in butt contact with longitudinal piezoelectric ultrasonic transducers, is examined. The method allows for prediction of a propagated waveform given a measured source waveform, together with the material properties of the cylinder. Alternatively, the source waveform can be extracted from measurement of the propagated waveform. The material properties required for implementation of the pulse propagation model are determined using guided wave phase velocity measurements. Hard tempered aluminum 1100 and 304 stainless steel wires, with 127, 305, and $406 \mu \mathrm{m}$ diam, were examined. In general, the drawn wires were found to behave as transversely isotropic media.
\end{abstract}

PACS numbers: 43.35.Pt, 43.20.Jr

\section{INTRODUCTION}

Historically, pulse propagation in thin cylindrical waveguides has been of interest for applications including the material testing of wires and fibers, ${ }^{1-3}$ ultrasonic delay lines, ${ }^{4}$ medical ultrasonics, ${ }^{5}$ and embedded sensor materials. ${ }^{6}$ Propagation of ultrasonic waves in cylinders has been the subject of several investigations, both analytical and experimental. ${ }^{7-20}$ In high-frequency and broadband applications, these cylinders may exhibit considerable dispersion when an acoustic pulse propagates through the waveguide. Here, dispersion is the dependence of acoustic guided wave phase velocity on frequency, resulting in distortion of the waveform of a transient plane-wave pulse depending on the axial location. This pulse distortion can make the interpretation of measured waveforms difficult. The present paper combines experimental measurements and analytical modeling to yield a quantitative and integrated approach to address the problem of pulse dispersion in cylindrical waveguides. The method presented also has significant relevance to the problem of material characterization of these waveguides.

\section{PULSE PROPAGATION}

While exact ${ }^{7-9}$ and approximate ${ }^{10,11}$ solutions have been used to study the axially symmetric guided wave phase velocity versus frequency relationship in solid cylindrical waveguides, and experimental dispersion measurements have corroborated the theory, ${ }^{12-15}$ few studies have been done to assess the capability these solutions yield for modeling actual pulse propagation. The earliest analytical solution, using exact theory, to the problem of axially symmetric pulse propagation in a cylindrical waveguide due to application of a transient end condition can be attributed to Skalak. ${ }^{\text {th }}$ A special solution was obtained to the problem of a solid isotropic cylinder with a velocity imparted uniformly to the cylinder end in the form of a step function. Subsequently, Folk et al. ${ }^{17}$ used exact theory to obtain a solution for a solid isotropic cylinder with arbitrary time harmonic mixed (combined displacement and stress) end boundary conditions. Moreover, an attempt was made by Folk et al. ${ }^{17}$ to experimentally validate the solution in terms of its ability to model pulse propagation. Studies by Miklowitz and Nisewanger, ${ }^{18}$ Hseih and Kolsky, ${ }^{19}$ and McNiven and Mengi ${ }^{20}$ have attempted to experimentally validate propagation models based on approximate theories. Only qualitative agreement between theoretical and measured waveforms was obtained in these studies. Furthermore, the pulses examined in all of these studies were dominated by lower-frequency propagation of the first longitudinal mode. The pulses, resulting from either ideal or modified (some finite rise time) step loading, essentially consist of a low-frequency leading head and a higher-frequency dispersive tail. While the current study also addresses first longitudinal mode propagation, the transient end conditions applied give rise to more varied dispersive pulse distortion. The frequency range considered extends from low frequency, where the fundamental tends toward nondispersiveness, to approaching the second longitudinal mode cutoff, where the fundamental becomes highly dispersive. An integrated analytical and experimental study of isotropic hollow cylinders using the same numerical method and similar measurement techniques ${ }^{21}$ involves the propagation of higher-order longitudinal modes.

\section{PROPERTY MEASUREMENT}

Because the dispersion of the guided waves depends on the elastic properties, cylindrical guided wave propagation has been used for the material characterization and testing of wires and fibers. Meeker and Meitzler ${ }^{22}$ suggested several 
methods for determining material properties by measuring acoustic guided wave velocities, and a thorough review of the subject has been given by Curtis. '

Principally, velocity measurements of the first torsional mode and the long wavelength limit of the first longitudinal mode have been used to obtain the shear and Young's moduli, respectively, for isotropic cylinders. ${ }^{1.2}$ The first axisymmetric torsional mode is nondispersive, propagating at the shear wave velocity $(G / \rho)^{1 / 2}$. In the long wavelength (low-frequency) limit, the velocity of the first longitudinal axisymmetric mode tends toward the bar velocity $(E / \rho)^{1 / 2}$. As the limit is approached, group and phase velocities tend toward a constant and equal value, indicating nondispersive behavior.

However, the drawing process in the manufacture of wires and fibers can affect the material microstructure, frequently causing the material to be anisotropic. ${ }^{1}$ Transverse isotropy, with five independent elastic constants, is considered to better represent the state of the material than isotropy. The five independent elastic properties for transversely isotropic media are taken as $E_{\mathrm{LL}}, E_{\mathrm{TT}}, G_{\mathrm{LT}}, G_{\mathrm{TT}}, \nu_{\mathrm{LT}}$, where $E, G$, and $\nu$ are Young's moduli, shear moduli, and poisson ratio, respectively, $L$ is longitudinal, and $T$ is transverse..$^{23}$ Moreover, the propagation of the fundamental longitudinal mode, within the frequency range considered, does not depend on the transversely isotropic property to which the lowest torsional mode is sensitive, $G_{\mathrm{LT}}$. Rather, the strongest dependence is on $E_{\mathrm{LL}}$ and $\nu_{\mathrm{LT}}$.

Longitudinal mode measurement to obtain $E_{\mathrm{LL}}$ can be problematic. When the velocity of the long wavelength limit is used, a transient pulse with sufficient low-frequency content is propagated and its first arrival measured. Since the longest wavelengths of the first longitudinal mode propagate with greatest velocity, this time-of-flight measurement yields the velocity of the long wavelength limit. However, the leading head of a dispersive pulse often rises very gradually making accurate assessment of when the first arrival occurs difficult, as discussed by Curtis, ${ }^{1}$ and evident in the lowfrequency experimental waveforms of other studies. ${ }^{17-20}$ Consequently, the measurement may be subject to some error. Instead, the time shift between the peak of the wave envelope of a propagated broadband pulse is often matched with that of the initial pulse. ${ }^{2}$ However, the frequency and wavelength of such a velocity measurement is not at all clear. $^{24}$

Alternatively, phase or group velocity of particular cylindrical guided wave modes can be measured over a range of frequency. Material properties are then varied to obtain correspondence between theoretical velocities and the dispersion measurements. As noted by Meeker and Meitzler, ${ }^{22}$ dispersion measurements of the first axially symmetric longitudinal mode are sufficient to find both elastic constants for isotropic media. As will be illustrated here, combination of such measurement with measures of first torsional mode velocity yields $E_{\mathrm{LL}}, \nu_{\mathrm{LT}}$, and $G_{\mathrm{LT}}$ for transversely isotropic media. The extent of the material anisotropy can then be gauged.

Phase velocity measurement methods are preferred to group velocity measurement methods since the wave propa-

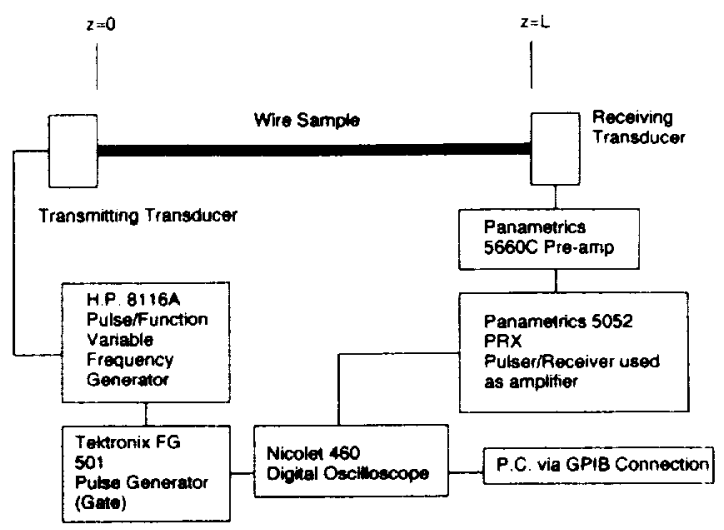

FIG. 1. Experimental apparatus.

gation is generally dispersive. Inherent difficulty in group velocity measurements for dispersive media has been noted. ${ }^{24.25}$ The problem stems from the distortion of the propagated waveform relative to the initially generated waveform and the resulting ambiguity in correlating the two different waveforms, and also, in determining the frequency for which the group velocity is being measured. Correspondingly, precision and accuracy for phase velocity measurement can be expected to exceed that obtained for group velocity measurement for dispersive pulse propagation. The scatter of group velocity measurements which commonly occurs for propagation in dispersive media can be seen in a recent study of sound in wires by Nicholson et al. ${ }^{15}$ The precision of the measurement and smoothness of the curve determined by these measurements is critical to accurate and repeatable material property characterization. The elastic properties determined from the phase velocity measurements are used here as input to the pulse propagation model.

\section{WAVE EXCITATION AND MEASUREMENT}

The method by which elastic pulses are launched into the wire specimens and measured after propagating the length of the sample is illustrated in Fig. 1. Similar techniques have been used in other studies of ultrasound propagation in wires. ${ }^{15}$ As shown in Fig. 1, matched pairs of piezoelectric longitudinal or shear wave transducers are placed in butt contact with each end of the wire and operate in pitch catch mode. For this study, $5 \mathrm{MHz}$ center frequency panametrics V109 (longitudinal) and V156 (shear) transducers were used. Glycerin was used as couplant to aid in sound transmission across the transducer/wire interface. The source or excitation transducer was provided a gated sine wave or toneburst electrical signal. Because of the thinness of the wires, the efficiency at which sound could be introduced into the waveguides was very low. Consequently, gain in the receiving system between 60 and $100 \mathrm{~dB}$ was required and signal averaging between 300 and 600 data records was performed to improve signal to noise. Signals were captured and averaged on a digital oscilloscope and processed on a PC.

With longitudinal wave transducers in use, the pulse launched in the medium would be composed primarily of the first axially symmetric longitudinal mode. However, depending on factors such as the wire thickness, excitation fre- 

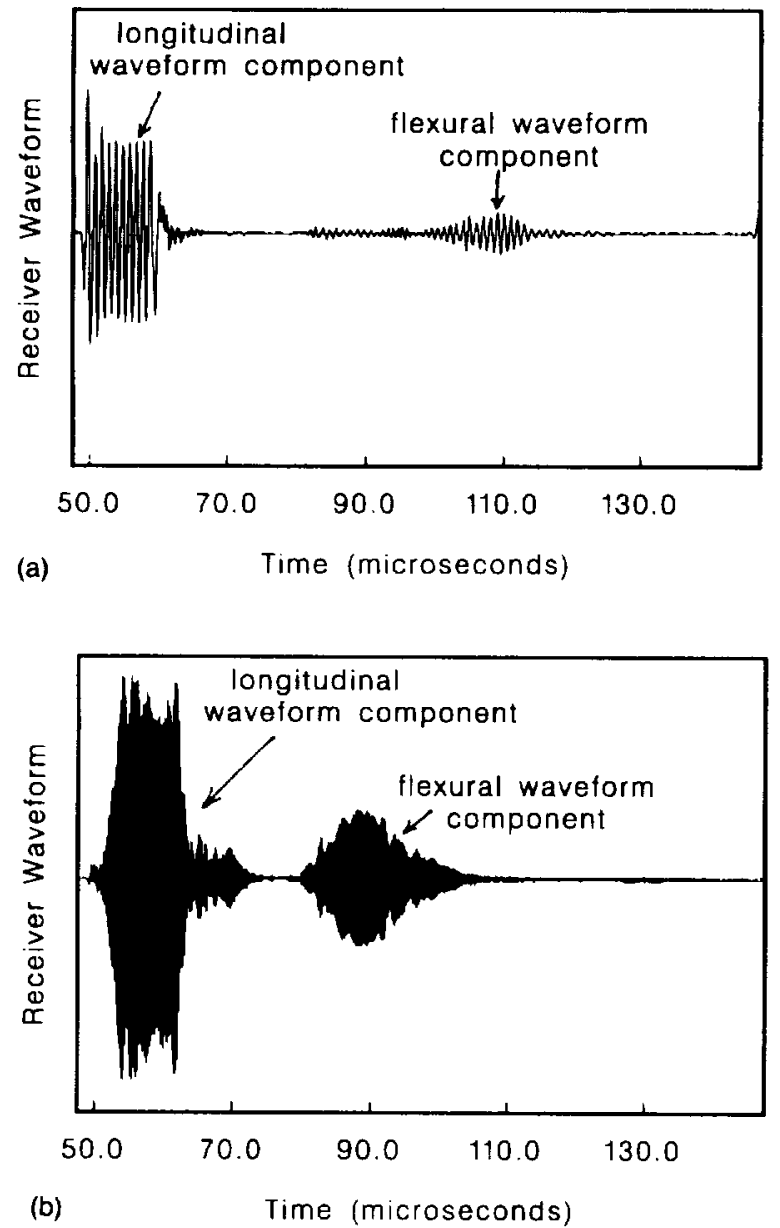

FIG. 2. Measured propagated pulse in a 24.76-cm-long, 12-mil-diam aluminum 1100 wire due to excitation of longitudinal piezoelectric source transducer with $1-\mathrm{MHz}$ (top) and $5-\mathrm{MHz}$ (bottom) tonebursts.

quency, couplant effects, and obviously the contact between source transducer and wire, the first-order flexural mode was also excited. In general, the flexural mode propagates much more slowly than the axisymmetric longitudinal mode. Consequently, the waveform due to each of the two modes can be temporally separated after some length of propagation. However, with increasing frequency, the dispersion of the waveguide is such that the first longitudinal mode is observed to propagate more slowly while the flexural mode is observed to propagate faster. Propagation of higher-frequency harmonics may result in overlap and interference between axially symmetric longitudinal and nonsymmetric flexural waveform components. The longitudinal and flexural components of typical measured waveforms for bursts with increasing center frequency are shown in Fig. 2. Longitudinal wave transducers are used for generating and receiving the wave. The waveform component associated with the flexural mode was eliminated through windowing for both the phase velocity measurements and the extraction of the source signal from a measured received waveform.

Torsional modes were excited by using shear wave transducers and intentionally placing only an edge of the wire end into contact with the source transducers face. As a result of such contact, both longitudinal and torsional axi-
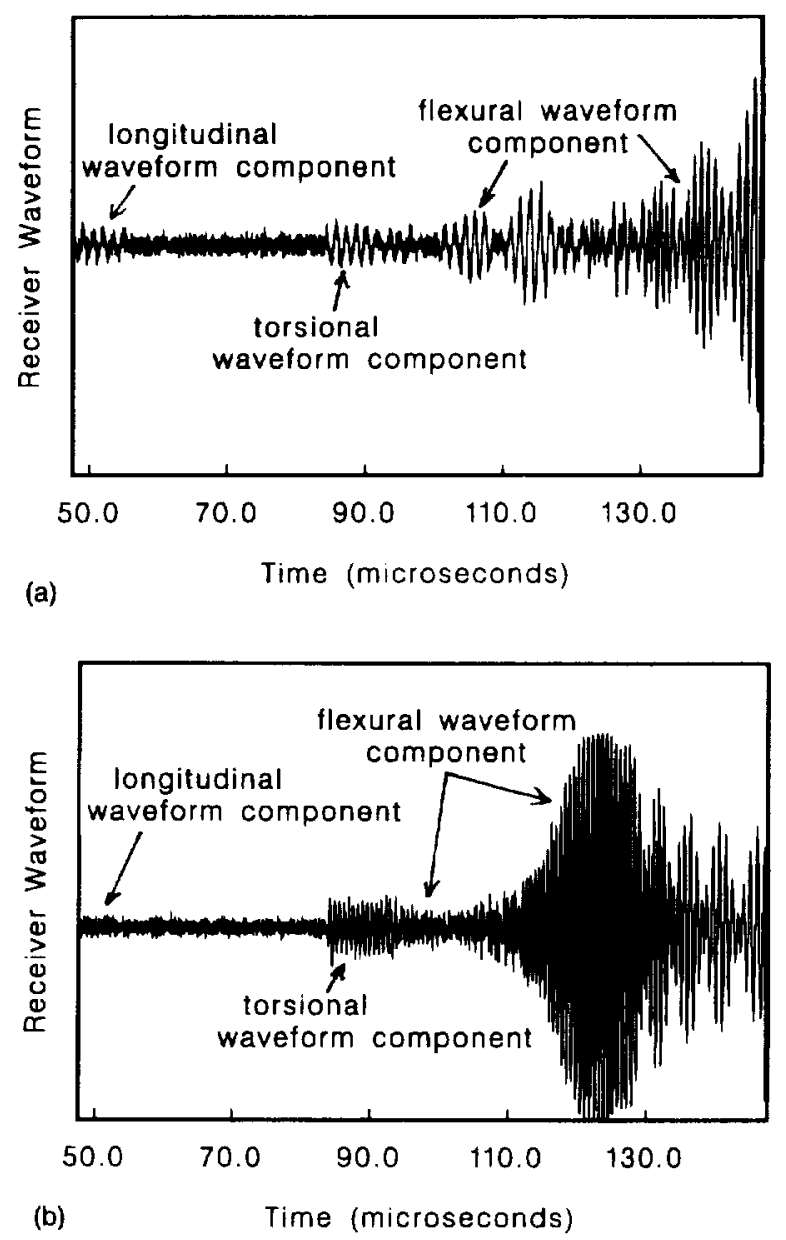

FIG. 3. Measured propagated pulse in a $25.22-\mathrm{cm}-\mathrm{long}$, 5-mil-diam 304 stainless steel wire due of excitation to shear piezoelectric source transducer with $700-\mathrm{kHz}$ (top) and $1.5-\mathrm{MHz}$ (bottom) tonchursts.

symmetric modes and at least first-order flexural modes were excited. By varying the frequency of the toneburst excitation of the shear wave transducer, and observing which wave packet remained fixed with regard to arrival and did not distort relative to the source waveform (nondispersive), the torsional wave packet could be identified. Torsional wave packets are shown for bursts of 0.7 and $1.5 \mathrm{MHz}$ in Fig. 3. Since dispersive first-order flexural waves are also excited, caution must be used as flexural waves will often propagate at similar velocities relative to torsional waves. Consequently there is overlap of the waveforms over broad ranges of frequency. In Fig. 3, the faster propagation of the flexural mode when a higher-frequency burst was used, results in the beginning of interference between the nondispersive torsional mode and the highly dispersive flexural mode.

Source measurements were performed by placing the matched transducers into face to face contact without the intervening wire. It should be noted that source measurement by this method is imperfect since it neglects all transducer/ wire interface effects. In particular, some variation in the behavior of the source transducer when electrically excited in unloaded (with wire contacting transducer face) as opposed to loaded (matched transducer face-to-face contact) condition could be anticipated. The effect of multiple reflections be- 
TABLE I. List of measured material properties.

\begin{tabular}{|c|c|c|c|c|}
\hline $\begin{array}{l}\text { Wire type (diameter } \\
\text { and density) }\end{array}$ & $E_{\mathrm{LL}}(\mathrm{GPa})$ & $\nu_{\mathrm{LT}}$ & $G_{\mathrm{LI}}(\mathrm{GPa})$ & $G_{\text {iso }}(\mathrm{GPa})$ \\
\hline \multicolumn{5}{|l|}{304 stainless steel } \\
\hline $\begin{array}{l}406 \mu \mathrm{m} \mathrm{(16} \mathrm{mil),} \\
7919 \mathrm{~kg} / \mathrm{m}^{3}\end{array}$ & 211 & 0.30 & 74.5 & 81 \\
\hline $\begin{array}{l}305 \mu \mathrm{m}(12 \mathrm{mil}) \\
7852 \mathrm{~kg} / \mathrm{m}^{3} \\
\text { (annealed) }\end{array}$ & 208 & 0.30 & 76.0 & 80 \\
\hline $\begin{array}{l}127 \mu \mathrm{m}(5 \mathrm{mil}) \\
7913 \mathrm{~kg} / \mathrm{m}^{3}\end{array}$ & 225 & 0.35 & 71.1 & 82 \\
\hline \multicolumn{5}{|l|}{ aluminum 1100} \\
\hline $\begin{array}{l}406 \mu \mathrm{m} \mathrm{(16} \mathrm{mil)} \\
2679 \mathrm{~kg}^{3} \mathrm{~m}^{3}\end{array}$ & 72.8 & 0.36 & 24.4 & 27 \\
\hline $\begin{array}{l}305 \mu \mathrm{m}(12 \mathrm{mil}) \\
2668 \mathrm{~kg} / \mathrm{m}^{3}\end{array}$ & 68.6 & 0.36 & 25.3 & 25 \\
\hline $\begin{array}{l}127 \mu \mathrm{m}(5 \mathrm{mil}) \\
2742 \mathrm{~kg} / \mathrm{m}^{3}\end{array}$ & 71.0 & 0.40 & 26.0 & 25 \\
\hline
\end{tabular}

increases. Most real dispersive pulses will have regions of frequency exhibiting nonphysically realizable phase $(\phi)$ information (i.e., corresponding to negative group velocity), often concomitant with low-magnitude complex amplitude $A(\omega)$. With a high density of phase jumps and even small regions of inaccurate phase information, the phase wraps become impossible to accurately track.

As noted previously, curve fitting of measured phase velocities of the first longitudinal axisymmetric mode through variation of material properties allows complete material property characterization for waveguides of isotropic media. ${ }^{22}$ The same method may be applied to transversely isotropic cylinders provided that $E_{\mathrm{LL}}$ and $\nu_{\mathrm{LT}}$ are taken as the independent properties to be varied in obtaining the curve fit. Phase velocity measurements from low frequency to frequencies evidencing significant dispersion (approaching the cutoff of the second longitudinal mode) are required for accurate determination of both material properties.

The lowest torsional axisymmetric mode is nondispersive, for both transversely isotropic and isotropic cylinders. This mode is sensitive only to $G_{\mathrm{LT}}$ for transverse isotropy. Once the torsional wavepacket is identified, its velocity is measured by cross-correlation. ${ }^{28}$

Table I summarizes the properties obtained in this manner for six different wire types investigated. The final column for each wire gives the shear modulus which would be required to support the conclusion of isotropy given the values of $E_{\mathrm{LL}}$ and $\nu_{\mathrm{LT}}$ determined by longitudinal mode measurement. Comparing this value of shear modulus with that obtained by torsional mode measurement is a measure of the material anisotropy and supports the conclusion that transverse isotropy better corresponds to the material behavior than isotropy, particularly for the steel wires. However, there is good agreement between solutions for the dispersion relations using either Pochhammer's exact solution for isotropic media taking $E$ and $v$ as independent properties, or using the numerical method described above and the complete set of five independent properties. The implication is that determination of $G_{\mathrm{LT}}$ was only of interest in verifying the anisotropy of the media, but this anisotropy has no significant effect on the first longitudinal mode behavior. Since the first longitudinal mode propagation was not sensitive to the exact values for $E_{\mathrm{TT}}$ and $G_{\mathrm{TT}}$, these were set equal to $E_{\mathrm{LL}}$ and $G_{\mathrm{LT}}$, respectively.

Figures 4 and 5 show measured longitudinal mode velocities and the curve fits obtained, for 16-mil $(406 \mu \mathrm{m})$ and 5-mil $(127 \mu \mathrm{m})$ diameter stainless steel wires, respectively. The entire frequency range for which data was taken was not necessarily used for each wire. The solid circles indicate phase velocity measurements at discrete frequencies used in the curve fit shown as a solid line. In Fig. 5, additional measurements not used in the curve fit are shown as squares.

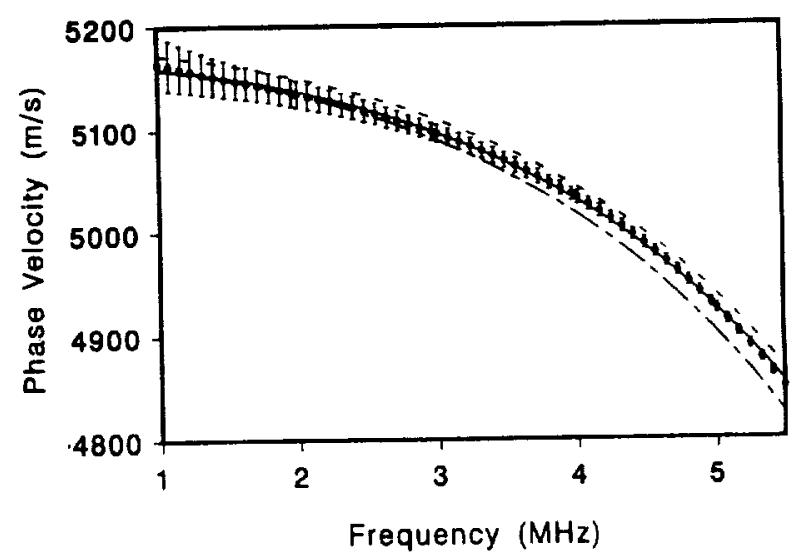

FIG. 4. Curve fit (solid line) of measured phase velocity (solid circles) to determine elastic properties for a 16-mil-diam 304 stainless steel wire. Analytical curves due to $0.5 \%$ increase in $E_{\mathrm{LL}}$ (dotted line) and $5 \%$ increase in $\nu$ (dot-dash line) are also shown. 


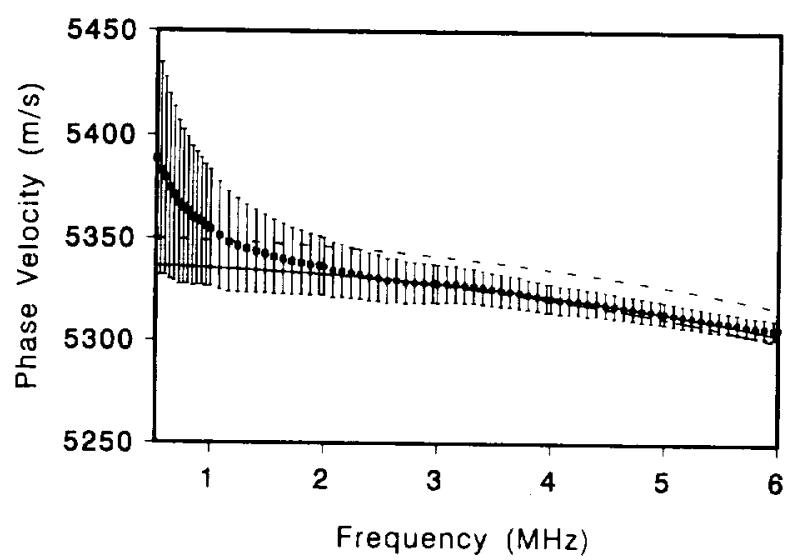

FIG. 5. Curve fit (solid line) of measured phase velocity (solid circles) to determine elastic properties for a 5-mil-diam 304 stainless steel wire. Analytical curves due to $0.5 \%$ increase in $E_{\mathrm{LI}}$ (dotted line) and $5 \%$ increase in $\nu$ (dot-dash line) are also shown.

Additional information regarding error analysis and accuracy is also conveyed and requires some explanation.

The appearance of the measured dispersion at lower frequencies in Fig. 5 does not correspond to the first mode dispersion for any elastic cylinder. Such measurement anomaly was much more significant for the smaller diameter $(127 \mu \mathrm{m})$ wires tested. The frequency dependences of transmission through both of the transducer/wire interfaces, which has been neglected, may plausibly be influenced by the wire diameter.

In general, several other factors may contribute to measurement error. First, the source waveform may be inadequately characterized, due to the difference in source transducer loadings between source measurement and transmission into the wire specimen. Furthermore, at the receiving end of the wire, some of the wave incident on the transducer is reflected, which may also give rise to some phase shift. The frequency difference between lowermeasurement frequencies and the resonant frequency of the transducer $(5 \mathrm{MHz})$ may exacerbate these problems.

If any resulting phase shift error at the pulse center frequency does not exceed some maximum value, say $\pi / 2$, and the determination of $N$ remains unambiguous, it is clear from Eq. (6a) that the phase velocity measurement becomes more accurate as frequency increases. The actual phase $(\phi)$ measurement becomes increasingly small relative to the quantity $2 \pi N$ in the denominator of Eq. (6a). The error bars of Figs. 4 and 5 indicate the change in measured phase velocity which would result from a $\pm \pi / 2$ shift in $\phi$.

Because of the apparent systematic error which occurred at lower frequencies for the 5-mil steel wire (Fig. 5), not all of the dispersive data available was used in the curve fit. That the analytical curve fit falls within the range of the error bars of the unused measurements for a $\pi / 2$ maximum error in $\phi$, indicates that the phase shift error corresponds to less than a quarter of a wavelength. For the 16-mil diameter stainless steel wire (Fig. 4), measurements beyond $5 \mathrm{MHz}$ were not used because of overlap and interference between longitudinal and flexural waves, as discussed previously.

The dashed lines of Figs. 4 and 5 show the alteration of
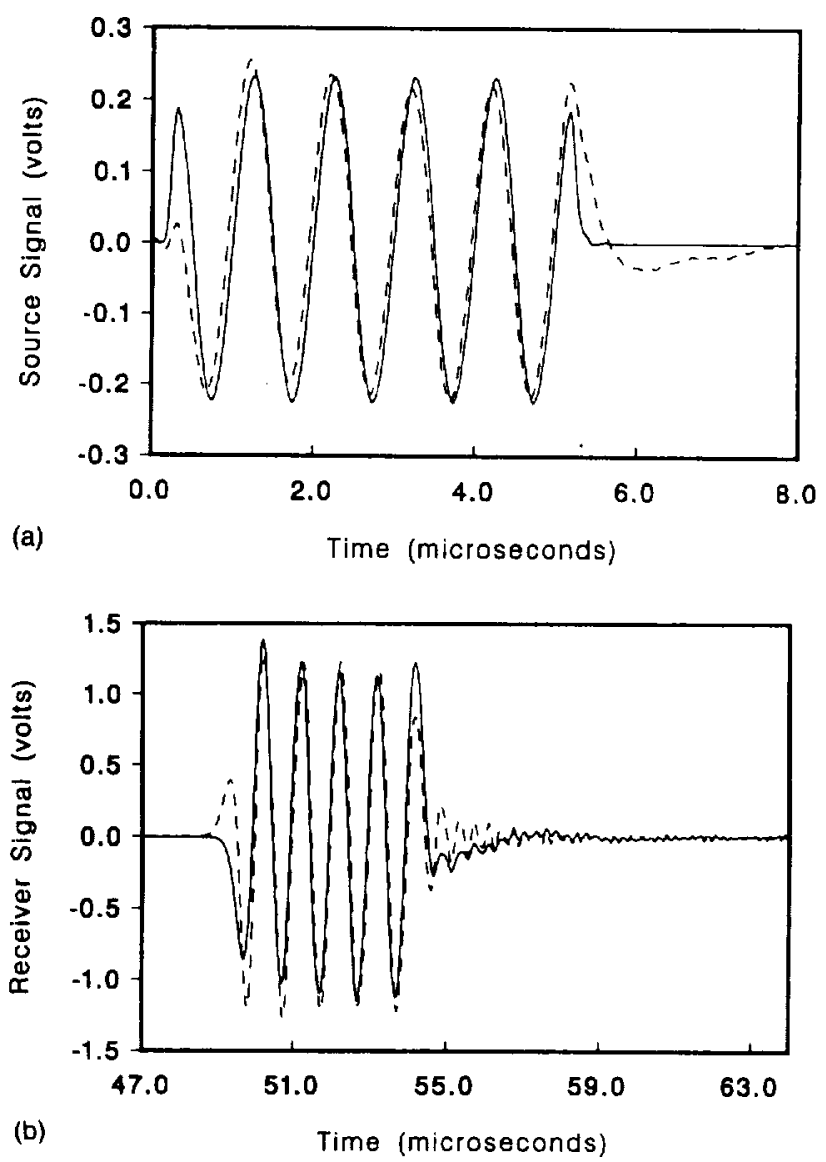

FIG. 6. Pulse propagation of a 1-MHz toneburst in 25.27-cm-long, 16-mildiam 304 stainless steel wire; (a) measured (solid line) and analytically extracted (dashed line) source waveforms, (b) measured (solid line) and analytically predicted (dashed line) receiver waveforms.

the analytically determined curves due to $0.5 \%$ and $5 \%$ variations in $E_{\mathrm{LL}}$ and $\nu_{\mathrm{LT}}$, respectively. In comparing Figs. 4 and 5 , specifically the dashed and solid curves in each figure, it is apparent that in order to determine $\nu_{\mathrm{LT}}$ accurately, data associated with the increased dispersion at higher frequency, approaching the second longitudinal mode cutoff, must be available. Consequently, accuracy of the determination of $\nu_{\mathrm{LT}}$ for the thicker wire was judged to be improved (i.e., the second mode cutoff is at much higher frequency for the thinner wire). The dispersion of the fundamental mode, as frequency and wire diameter to wavelength ratio increase, has been associated with the significance of radial inertia. ${ }^{10,11} \mathrm{~A}$ conservative accuracy estimate of better than $0.5 \%$ for $E_{\mathrm{LL}}$ and $5 \%$ for $\nu_{\mathrm{LT}}$ is claimed when adequate dispersion measurements are available.

\section{COMPARISON OF EXPERIMENTALLY MEASURED AND MODELED PULSE PROPAGATION}

Comparison of experimentally measured receiver waveforms with analytically predicted responses, due to various measured sources, for the 16-mil steel wire, are shown in Figs. 6(b), 7(b), 8(b), and 9(b). The measured sources engendering these responses are shown in Figs. 6(a), 7(a), 8(a), and 9(a) together with the source waveform extracted from the aforementioned measured receiver waveforms. The analyti- 


\section{ACKNOWLEDGMENTS}

This work was supported in part by NASA Grant NAG1-1390 and the George M. Low Fellowship, National Space Club.

${ }^{1}$ G. J. Curtis, "Wave Propagation Techniques in Determining the Dynamic Elastic Properties of Wires and Fibers," in Ulrrasonic Testing, NonConventional Testing Techniques, edited by J. Szilard (Wiley, New York, 1982), pp. 555-598.

${ }^{2}$ L. C. Lynnworth, "Ultrasonic Measurement of Elastic Moduli in Slender Specimens Using Extensional and Torsional Wave Pulses," J. Test. Eval. 1, $119-125$ (1973).

"J. W. Ballou and S. Silverman, "Young's Modulus and Fibers and Films by Sound Velocity," J. Acoust. Soc. Am. 16, 113-119 (1944).

"J. E. May, "Guided Wave Ultrasonic Delay Lines," in Physical Acoustics, edited by W. P. Mason (Academic, New York, 1964), Vol. IA, pp. 417 . 483.

${ }^{5}$ N. C. Nicholson and McDicken, W. N., "Waveguides in Medical Ultrasonics," Ultrasonics 26, 27-30 (1988).

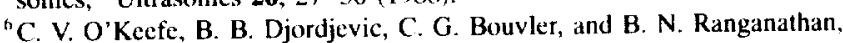
"In-Situ Sensors and Structural Health Monitoring Applications," presented at Review of Progress in Quantitative NDE, 19-24 July 1992, San Diego, CA.

${ }^{7}$ L. Pochhammer, "Ueber dic Fortpfanzungsgeshwindigkeiten Kleiner Schwingungen in einum unbregrenzien isotropen Kreiszylinder," Z. Math. 81, 324-336 (1876).

${ }^{*}$ M. Onoe, H. D. McNiven, and R. D. Mindlin, "Dispersion of Axially Symmetric Waves in Elastic Rods," J. Appl. Mech. 29, $729-734$ (1962).

${ }^{9} 1$. Mirsky, "Wave Propagation in Transversely Isotropic Circular Cylinders Part 1: Theory, Part 1I: Numerical Results," J. Acoust. Soc. Am. 37, 10161026 (1965).

${ }^{10}$ R. D. Mindlin and H. D. McNiven, "Axially Symmetric Waves in Elastic Rods," J. Appl. Mech. 27, 145-151 (1960)).

${ }^{11}$ R. D. Mindlin and G. Herrmann. "A One-Dimensional Theory of Compressional Waves in an Elastic Rod," in Proceedings' of the First U. S National Congress of Applied Mechanics, June 1951 (ASME, New York. 1952), pp. $187-191$.

${ }^{12}$ G. E. Hudson, "Dispersion of Elastic Waves in Solid Circular Cylinders," Phys. Rev. 63, 46-51 (1943).

${ }_{1.3}$ L. Y. Tu, J. N. Brennan, and J. A. Sauer, "Dispersion of Ultrasonic Pulse Velocity in Cylindrical Rods," J. Acoust. Soc. Am. 27, 550-555 (1955).
${ }^{14}$ J. Zemanek, "An Experimental and Theoretical Investigation of Elastic Wave Propagation in Cylinders," Doctoral dissertation, University of California, Los Angeles, CA (1962).

${ }^{15}$ N. C. Nicholson, W. N. McDicken, and T. Anderson, "Waveguides in Medical Ultrasonics: An Experimental Study of Mode Propagation," Ultrasonics 27, 101-106 (1989).

${ }^{16}$ R. Skalak, "Longitudinal Impact of Semi-Infinite Circular Elastic Bars," J. Appl. Mech. 24, 59-64 (1957).

${ }^{17}$ R. Folk, G. Fox, C. A. Shook, and C. W. Curtis, "Elastic Strain Produced by Sudden Application of Pressure to One End of a Cylindrical Bar," J. Acoust. Soc. Am. 30, 552-563 (1958).

${ }^{18} \mathrm{~J}$. Miklowitz and C. R. Nisewanger, "The Propagation of Compressional Waves in a Dispersive Elastic Rod, Part II-Experimental Results and Comparison with Theory," J. Appl. Mech. 24, 240-244 (1957).

${ }^{14}$ D. Y. Hsieh and H. Kolsky, "An Experimental Study of Pulse Propagation in Elastic Cylinders," Proc. Phys. Soc. 71, 608-612 (1958).

${ }^{2}$ H. D. McNiven and Y. Mengi, "Experimental Assessment of the MindlinMcNiven Rod Theory," J. Acoust. Soc. Am. 62, 589-594 (1977).

${ }^{21}$ T. Kohl, W. P. Rogers, and S. K. Datta, "Dispersive Pulse Propagation and Material Characterization for Hollow Cylindrical Waveguides," in Scientific and Engineering Aspects of Nondestructive Evaluation, Proceedings of 1993 ASME Pressure Vessels and Piping Division Conference, NDE Engineering Division, edited by M. N. Srinivasan (ASME, New York, 1993).

22T. R. Meeker and A. H. Meitzler, "Guided Wave Propagation," in Physical Acoustics, edited by W. P. Mason (Academic, New York, 1964), Vol. 1A, pp. $111-167$

${ }^{23}$ R. M. Christensen, Mechanics of Composite Materials (Wiley, New York, 1479), pp. 73-78.

${ }^{24}$ E. M. Young, "Discussion of Time Delay in Reference to Electrical Waves," IRE Trans. Ulirason. Eng. UE-9, 13-21 (1962).

${ }^{25}$ W. Sachse and Y. H. Pao, "On the Determination of Phase and Group Velocities of Dispersive Waves in Solids," J. Appl. Phys. 49, 4320-4.327 (1978).

"T. W. Kohl, S. K. Datta, and A.H. Shah, "Axially Symmetric Pulse Propagation in Semi-Infinite Hollow Cylinders," AIAA J. 30, 1617-1624 (1992).

${ }^{27}$ K. H. Huang and S. B. Dong, "Propagating Waves and Edge Vibrations in Anisotropic Composite Cylinders," J. Sound Vib. 96, 363-379 (1984).

${ }^{2 x}$ D. R. Mull, H. E. Kautz, and A. Vary, "Measurement of Ultrasonic Velocity Using Phase-Slope and Cross-Correlation Methods," Mater. Eval. 43, $1455-1460)(1985)$. 